\title{
Suppression of irrelevant activation in the horizontal and vertical Simon task differs quantitatively not qualitatively
}

\author{
Lisa Töbel ${ }^{\mathrm{a}, *}$, Ronald Hübner ${ }^{\mathrm{a}}$, Birgit Stürmer ${ }^{\mathrm{b}}$ \\ a Universität Konstanz, Germany \\ b International Psychoanalytic University Berlin, Germany
}

\begin{abstract}
A B S T R A C T
The Simon effect is usually explained by the assumption that the irrelevant stimulus location automatically activates the corresponding response. In the case of incongruent stimulus-response assignments automatically activated responses therefore have to be suppressed to ensure correct responses. This account, however, has been called into question for other than horizontally arranged visual Simon tasks. We investigated whether there is a qualitative or quantitative difference in suppression of irrelevant activation between horizontally and vertically arranged Simon tasks, using delta-function analyses. Sequential analyses revealed suppression after incongruent trials in both tasks, supporting the idea of a quantitative rather than a qualitative difference between the tasks. We conclude that automatic response activation is weaker in vertical tasks resulting in lower inhibitory demands as compared to horizontal tasks.
\end{abstract}

\section{Introduction}

In everyday life conflicting response tendencies have to be solved to guarantee accurate behavior. A widely applied paradigm to investigate conflict control is the Simon task introduced by Simon and Rudell (1967). In its visual version, the task requires categorizing a pre-defined non-spatial stimulus feature (e.g., color or shape) and to signal the result by a spatial choice response (e.g., left or right button press). In addition to the relevant non-spatial stimulus feature, however, the stimulus also has an irrelevant spatial dimension (e.g., it appears left or right of fixation) that overlaps with the spatial dimension of the responses. Usually, participants respond faster and more accurately to congruent stimuli (i.e. when stimulus location corresponds to the side of the required response) than to incongruent stimuli (i.e. when the stimulus appears opposite to the side of the correct response). The difference in response time (RT) and error rate between congruent and incongruent trials is called the Simon effect (see Hommel, 2011 for an overview).

Although the Simon task has been investigated extensively, its origin is still not fully understood. A widely accepted basic account, however, is the dual-route model which assumes that information flows from perception to the response along two routes, a conditional and an

\footnotetext{
* Corresponding author at: Universität Konstanz, Fachbereich Psychologie, Fach D29, D-78457 Konstanz, Germany.

E-mail address: 1toebel@gmail.com (L. Töbel).
}

unconditional one (De Jong, Liang, \& Lauber, 1994; Kornblum, Hasbroucq, \& Osman, 1990). Whereas task relevant stimulus information has to be translated to the correct response along the conditional route, irrelevant location information automatically activates the corresponding response via the unconditional route.

That stimulus location automatically affects response selection in the Simon task is supported by the characteristic of the so-called delta functions for the latencies of correct responses (delta functions for RT) and of delta functions for accuracy, which reflect how the Simon effect varies with RT in the latencies and error rates, respectively. In delta functions for accuracy, the size of the congruence effect for the fast responses is an indicator of the strength of automatic response activation (Ridderinkhof, 2002b). Usually, the Simon effect in error rates is relatively large for fast responses and decreases quickly towards zero, as late responses are highly accurate. This effect indicates fast automatic response activation by the location of the stimulus.

Also for correct responses, the Simon effect is relatively large for fast responses and decreases with increasing RT (see for example De Jong et al., 1994), hence, the delta function for RT has a negative slope. Interestingly, this effect is contrary to what one would expect, as the variance of RT is positively correlated with the mean RT (Wagenmakers \& Brown, 2007), which would usually result in a positively sloped delta function (Pratte, Rouder, Morey, \& Feng, 2010). The fact that the Simon effect decreases with RT for correct responses has been explained by suppression of automatic response activation that builds up gradually with time 
(De Jong et al., 1994; Ridderinkhof, 2002a; Ridderinkhof, van den Wildenberg, Wijnen, \& Burle, 2004).

Automatic activation of the corresponding response by stimulus location, however, has been called into question as a general account of the Simon effect, primarily, because the effect differs in its dynamics depending on whether stimulus location varies along the horizontal or the vertical meridian (e.g., Wascher, Schatz, Kuder, \& Verleger, 2001). The relative positions of the response buttons in both tasks correspond to that of the stimulus locations (e.g., when the stimuli vary along the vertical meridian, the participants are supposed to respond with an upper and a lower button). Thus, the spatial dimension of the stimulus always overlaps with that of the responses. Whereas negatively sloped delta functions for RT are found for the horizontal Simon task (HST), the vertical Simon task (VST) usually produces constant or even positively sloped delta functions for RT (e.g., Proctor, Vu, \& Nicoletti, 2003; Wascher et al., 2001). For a detailed review on differences in delta functions for RT between HST, VST, and other variants of the Simon tasks see Proctor, Miles, and Baroni (2011).

In view of such results Wascher et al. (2001) hypothesized that stimulus location does not activate the spatially corresponding response automatically in every case, but rather specific conditions have to be met. Such a condition is given, for instance, when a visual stimulus is processed in the same cerebral hemisphere as the response primed by the location of the stimulus. Obviously, this is the case in the standard HST. If such favorable conditions are not met, as in the VST, stimulus information is transmitted solely via the conditional route. Thus, the Simon effect in a VST is seen to arise during stimulus-response translation. Because no automatic response activation that triggers suppression in a VST is elicited, the Simon effect does not decrease but remains constant or even increases with RT. Several studies reported declining delta functions for RT exclusively in the standard HST (e.g., Vallesi \& Umiltà, 2009; Wiegand \& Wascher, 2005, 2007), supporting the idea of Wascher et al. (2001).

There are, however, some reports of declining delta functions for RT in Simon tasks when stimulus processing and response activation were not located in the same hemisphere. For instance, it has been shown that the Simon effect also decreases with increasing RT in a HST when responses are given with fingers of the same hand (Hübner \& Mishra, 2013; Proctor $\&$ Vu, 2010), by responding with saccadic eye movements (Wijnen \& Ridderinkhof, 2007), or by moving one hand to the left or to the right (Buetti \& Kerzel, 2008). Furthermore, in some studies the Simon effect also decreased with RT for the VST, e.g., when the stimulus-response mapping was randomized (Wiegand \& Wascher, 2007). These studies support the alternative hypothesis that HST and VST merely differ quantitatively. Rubichi, Nicoletti, and Umiltà (2005) suggested that location-induced activation is also present in the VST, but to a lesser degree than in the HST. Tsai, Chen, Jang, and Liao (2013) reported that the cortical magnification factor is smaller for a distance along the vertical axis as compared to the same distance along the horizontal axis. Possibly the larger representation on the visual cortex of the same distance in the HST as compared to the VST results in stronger automatic response activation in the former case. If automatic response activation is generally lower in VST, inhibitory demands are lower as well and less suppression is necessary, so that the Simon effect does not decrease with RT. According to this idea we should find suppression of irrelevant activation in the VST, when the inhibitory demand is relatively high.

The hypothesis that both tasks differ in their response activation is, however, also supported by EEG analyses (Vallesi, Mapelli, Schiff, Amodio, \& Umilta, 2005) and by showing different training effects in both tasks (Vu, 2007). More information about automatic response activation in both tasks could be gathered by also considering delta functions for accuracy, which are hardly reported in the literature on the VST. Fast error responses are influenced especially strongly by stimulus location and are not included in the delta functions for RTs, because only correct responses are included. Thus, the strength of automatic response activation is more reflected in the delta functions for accuracy, which, therefore, can be an important source of information for investigating the differences and similarities between the VST and the HST.

With the present study we aimed at further investigating whether the horizontal and vertical versions of the Simon task differ quantitatively or qualitatively. We assumed that any demonstration of suppression in the VST strengthens the idea of automatic response activation. However, automatic response activation should also be observed more directly by considering delta functions for accuracy. But how can we study suppression of irrelevant activation in the VST if there is no decrease of the Simon effect with RT? Our idea was to consider a variable that is well-known to modulate suppression and to see whether it affects performance in the VST in the same way as in the HST. The examined variable was the previous-trial congruency. In numerous studies it has been shown that previous-trial congruency has a substantial impact on the Simon effect (e.g., Stürmer, Leuthold, Soetens, Schröter, \& Sommer, 2002; see Egner, 2007 for a review). More specifically, the Simon effect is usually smaller after an incongruent than after a congruent trial. This modulation has been explained by conflict adaptation (Botvinick, Braver, Barch, Carter, \& Cohen, 2001) that serves for reducing the effects of irrelevant information after a conflict has been detected (Stürmer \& Leuthold, 2003; Stürmer et al., 2002; see Hommel, 2004, for an alternative account).

For the HST, Ridderinkhof (2002a) has shown that the Simon effect is generally reduced in trials following an incongruent one, and in addition the slope of the corresponding delta function for RT is more negative. This suggests that suppression of automatic response activation is increased after experiencing a response selection conflict in the previous trial. If one could show that previous-trial congruency also modulates the slope of the delta function for RT in the VST, then this could be taken as an indicator of automatic response activation in this task. Furthermore, if there is suppression of automatically induced responses in the VST, one should also observe a significantly declining delta function for RT, at least after incongruent trials.

Indeed, Stürmer et al. (2002) already observed that in a VST the Simon effect was reduced after incongruent trials compared to congruent ones and the slopes of the respective delta function for RT decreased, too. Unfortunately, Stürmer et al. (2002) did not test whether the reduction was significant, nor did they include a HST for comparison. They also did not exclude direct trial repetitions in the graph, which are supposedly confounded with conflict adaptation, as the response in these trials is usually very fast (Mayr, Awh, \& Laurey, 2003). In the present study we therefore conducted behavioral experiments to test whether we can find a similar reduction of the Simon effect in the VST with RT. In Experiment 1 we also included a HST in addition to the VST.

\section{Experiment 1}

In our first experiment we used a similar method as Stürmer et al. (2002). However, additionally to the VST we included a HST to compare the Simon effect and its modulation by previous-trial congruency between the two tasks. Because in the pilot studies we found that suppression decreases in the HST with the duration of the experiment, we used a between-participants design. A comparison of a balanced withinparticipant design could have been problematic, as the first and the second half of a test block are possibly not comparable.

Whereas predictions on mean Simon effects are not easy, because two opposing factors are at play (suppression and automatic response activation), clear predictions can be made for the delta functions. For accuracy we expected a larger Simon effect in the first quintile in the HST compared to the VST because automatic response activation is supposedly stronger (Rubichi et al., 2005). Further, we expected weaker automatic response activation in both tasks after incongruent trials. In the HST the delta function for RT should be negatively sloped, whereas it should be flat or even positively sloped in the VST. Critically, if these differences in the delta functions for RT merely reflect a quantitative difference between suppression of irrelevant response activation in the two 
tasks, then the modulation of suppression, and therefore of the delta functions for RT, by previous-trial congruency should be similar.

\subsection{Method}

\subsubsection{Participants}

16 participants (age range: $21-28$ years, mean: 23.7 years; 5 men) performed the HST and 19 participants (age range: 18-32 years, mean: 22.0 years; 6 men) performed the VST in a betweenparticipants design. All were recruited at the University of Konstanz and had normal or corrected-to-normal vision. Participants were paid $(8 € / \mathrm{h})$ for their participation or received course credits.

\subsubsection{Apparatus and stimuli}

The stimulus set consisted of a diamond and a square $\left(0.66^{\circ} \times 0.66^{\circ}\right)$. Each stimulus was presented in white against a black background on an $18^{\prime \prime}$ color-monitor with a resolution of $1280 \times 1024$ pixels, and a refresh rate of $60 \mathrm{~Hz}$. In the HST, the stimulus appeared either left or right $\left(1.2^{\circ}\right)$ from a central fixation cross $\left(0.23^{\circ} \times 0.23^{\circ}\right)$, whereas in the VST the stimulus appeared above or below the cross $\left(1.2^{\circ}\right)$. Participants had a viewing distance of about $45 \mathrm{~cm}$ from the screen. Their responses were recorded from the left and right control keys on a keyboard (HST), or from the down arrow and the Pos1 key (VST) on a QWERTZ keyboard. Obviously, the distance between the keys differed across the tasks. The reason was that we wanted to allow a rather natural limb position in both tasks. However, we expected that this difference will not substantially affect the slope of the delta functions for RT. For instance, a typical negatively sloped delta function for RT can be found in the HST even for responses with the fingers of the same hand (e.g., Hübner \& Mishra, 2013).

\subsubsection{Procedure}

The fixation cross was permanently present. Stimuli were shown for $100 \mathrm{~ms}$ and the next trial started about $1050 \mathrm{~ms}$ (varied randomly between 1000 and $1100 \mathrm{~ms}$ ) after the response. Participants had to decide as fast and as accurately as possible whether the stimulus was a diamond or a square. In the HST, half of the participants responded with the left key towards the diamond and with the right key towards the square; the other half got the opposite mapping. In the VST, half of the participants pressed the upper key with their left index finger and the lower key with their right index finger. For the other half the fingerto-key mapping was reversed. The stimulus-to-key mapping was also balanced across participants. Each task consisted of 17 test blocks, containing 80 trials; the first 20 trials served as practice trials. Thus, in total, there were 1340 trials and the experiment lasted about $50 \mathrm{~min}$.

\subsection{Results}

\subsubsection{Response times}

Only correct responses with latencies between $100 \mathrm{~ms}$ and $2000 \mathrm{~ms}$ were analyzed. Trials preceded by an error were excluded.

A three-factor ANOVA was computed, including the withinparticipant factors congruency (congruent or incongruent) and previoustrial congruency (congruent or incongruent), and the betweenparticipants factor task (HST or VST). It revealed a significant main effect of congruency $[F(1,33)=276.43, p<0.001]$, meaning that participants were faster in congruent ( $403 \mathrm{~ms}$ ) than in incongruent trials $(427 \mathrm{~ms})$. However, the interaction between congruency and task was also significant $[F(1,33)=6.87, p<0.05]$, indicating that the Simon effect was larger in the VST as compared to the HST (VST: $28 \mathrm{~ms}$; HST: $20 \mathrm{~ms}$ ). Moreover, the interaction between congruency and previous-trial congruency was significant $[F(1,33)=218.77, p<0.001]$, indicating a larger Simon effect after a congruent ( $50 \mathrm{~ms}$ ) than after an incongruent trial $(-2 \mathrm{~ms})$. The three-way interaction was not significant $[F(1,33)<1]$, indicating that the modulation of the Simon effect by previous-trial congruency was not different between the two tasks.

\subsubsection{Error rates}

The mean error rate was 7.39\%. An ANOVA of the same type as for the RT was also computed for the error rates. ${ }^{1}$ It revealed a significant main effect of congruency $[F(1,33)=76.75, p<0.001]$. Participants responded more correctly on congruent trials (congruent: $4.64 \%$; incongruent: $10.16 \%$ ). The main effect of previous-trial congruency was also significant $[F(1,33)=51.53, p<0.001]$. Participants made more errors after congruent trials (congruent: 8.64\%; incongruent: 6.03\%). The factor task did not reach significance $[F(1,33)=2.56, p=0.12]$. There was also a reliable interaction between congruency and previous-trial congruency $[F(1,33)=99.58, p<0.001]$, indicating a larger Simon effect after congruent (11.66\%) than after incongruent trials ( $-1.16 \%)$. The three-way interaction was not significant $[F(1,33)<1]$, suggesting that the Simon effect in the error rates was similarly modulated by previous-trial congruency in the two tasks.

\subsubsection{Distributional analyses}

Cumulative distribution functions (CDFs) for the latencies of correct responses were computed for each congruency condition (congruent, incongruent) for all trials (overall function) and for each previous-trial condition (congruent, incongruent; exact trial repetitions were excluded in the previous-trial congruency analyses) by quintile-averaging $(.1, .3$, $.5, .7$, and .9, see Ratcliff, 1979). The CDFs conditioned on previous-trial congruency are shown in Fig. 1. For calculating the delta functions for the RTs presented in Fig. 2, the quintile RTs for congruent trials were subtracted from those for incongruent trials and related to the respective mean quintile RTs.

Delta functions for accuracy were constructed by first calculating the conditional accuracy functions (CAFs) shown in Fig. 3 (only CAFs conditioned on previous-trial congruency are depicted). That is, all data were sorted into quintiles and the accuracy value was calculated for each quintile and condition separately. The accuracy values for each quintile for congruent trials were then subtracted from those for incongruent trials and were related to the respective mean quintile RTs. Delta functions for accuracy are depicted in Fig. 4.

\subsubsection{Response times}

As can be seen by inspecting the overall delta functions for RT in Fig. 2, the Simon effect decreased in the HST and slightly increased in the VST. If we take previous-trial congruency into account, however, the functions show a different pattern of results. In the HST, the Simon effect was relatively constant across RT after a congruent trial, but decreased after an incongruent trial. In the VST the Simon effect increased with RT after a congruent trial, but slightly decreased with RT after an incongruent trial.

In order to analyze the variation of the Simon effect with RT statistically, we subjected the cumulative distribution functions into a $2 \times 5 \times 2$ ANOVA with the within-participant factors congruency (congruent, incongruent) and quintile (1 to 5), and the between-participants factor task (HST, VST). ${ }^{2}$ We were not interested in the main effects, but merely in significant interactions with quintile. A significant interaction with the factor quintile would indicate that the congruence effect differed significantly between quintiles. If there is a decrease in the delta function, a significant interaction with quintile would indicate that the function has a significant negative slope. If the function is increasing, however, the same result would indicate a significant positive slope.

The analysis revealed a reliable three-way interaction between congruency, quintile, and task $[F(4,132)=11.16, p<0.001]$. It indicates

\footnotetext{
Analyses with arcsine transformed error rates revealed the same essential results.

2 Comparing the slopes of the delta functions for RT, estimated by least square regres-
} sion (Pratte et al., 2010) leads to the same conclusions. 

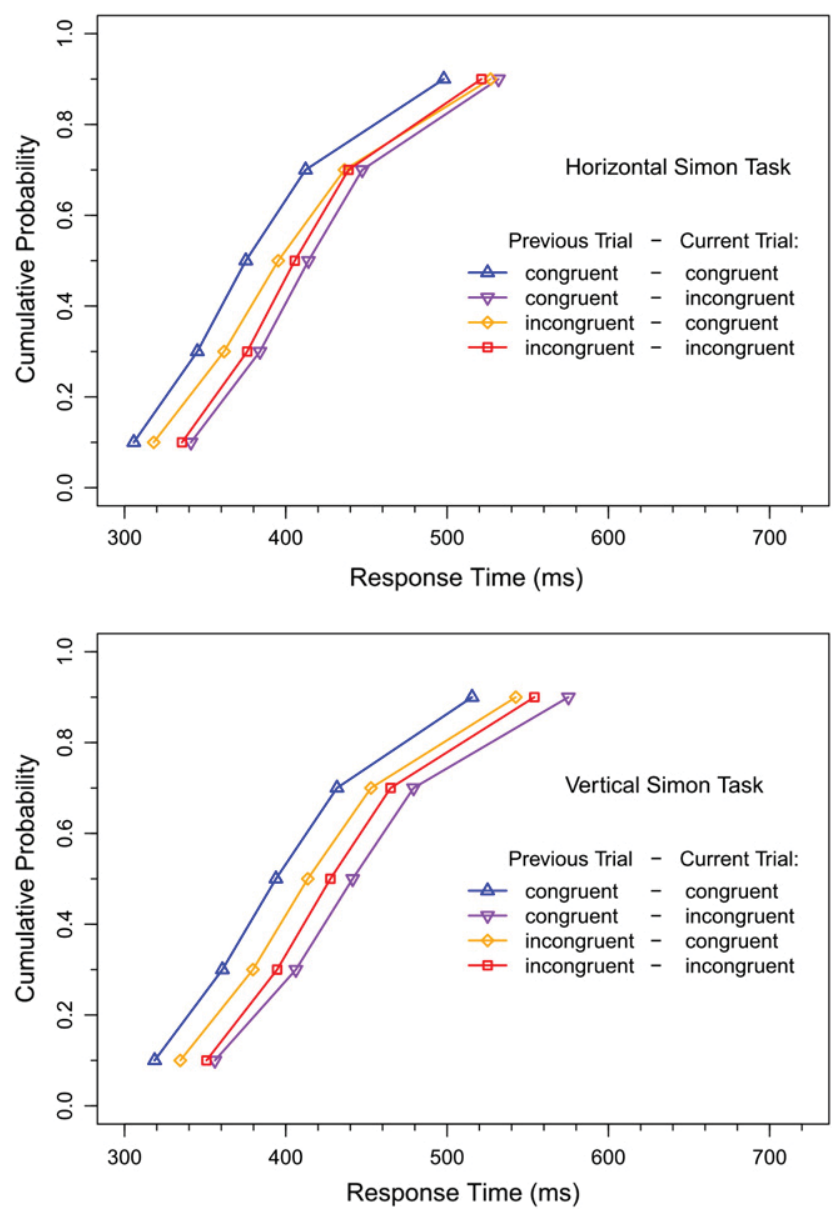

Fig. 1. CDFs for the HST (upper panel) and the VST (lower panel) of Experiment 1 . Only the specific functions conditioned on previous-trial congruency are depicted.

that the Simon effect varied differently with RT across quintiles in the two tasks. A separate analysis for the two tasks revealed a reliable two-way interaction between the factors congruency and quintile $[F(4,60)=3.95, p<0.01]$ in the HST, indicating that the Simon effect varied (decreased) with RT. The analogous interaction was also significant in the VST $[F(4,72)=10.88, p<0.001]$, where it indicates an increasing overall Simon effect, however.

In a further analysis we excluded direct stimulus repetitions and additionally included previous-trial congruency as a factor. Accordingly, we conducted another $2 \times 2 \times 5 \times 2$ ANOVA with the withinparticipant factors congruency, previous-trial congruency, and quintile, and the between-participants factor task. As in the previous analysis, there was a reliable three-way interaction between congruency, quintile and task $[F(4,132)=7.33, p<0.001]$. Most interestingly, though, the four-way interaction between all factors was far from significant $[F(4,132)<1]$, suggesting that the modulation of the Simon effect by previous-trial congruency did not differ between the HST and the VST. The small effect size $\left(\eta^{2}=0.007\right)$ of the interaction indicates that it presumably would not get significant even with an increased number of participants.

We applied the previous-trial congruency analyses separately for the VST, as it is of particular interest whether the delta function for RT declines in the VST. Accordingly, a $2 \times 2 \times 5$ ANOVA was calculated with the factors congruency, previous-trial congruency, and quintile. The analyses revealed a significant three-way interaction $[F(4,72)=6.63$, $p<0.001$ ], indicating that the slope of the delta function for responses after incongruent trials decreased, compared to that for responses after congruent trials. Further tests revealed that the increase of the Simon effect with RT after congruent trials was significant $[F(4,72)=9.17$,
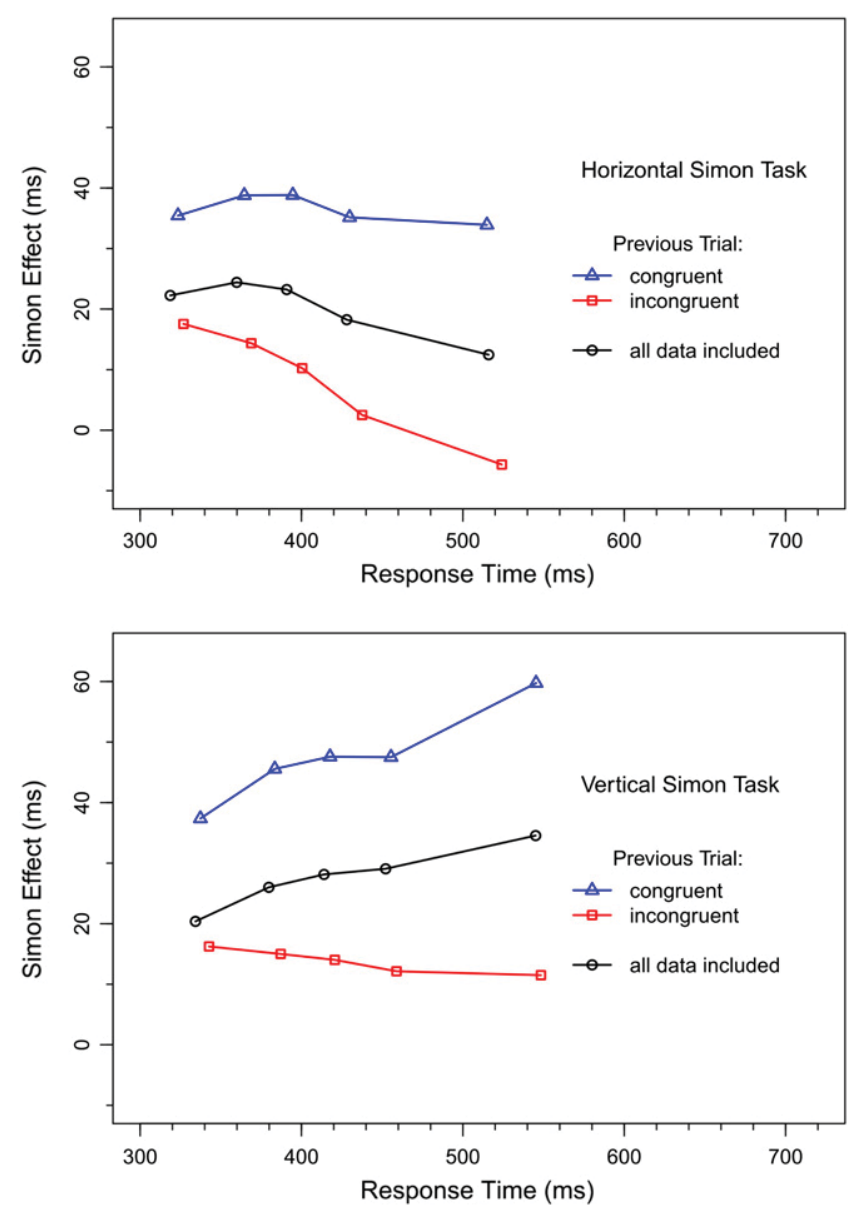

Fig. 2. Delta functions for the latencies of correct responses of the HST (upper panel) and the VST (lower panel) of Experiment 1. Overall functions (including all trials) as well as the specific functions conditioned on previous-trial congruency are depicted.

$p<0.001]$, whereas the Simon effect did not vary significantly for responses after incongruent trials $[F(4,72)<1]$.

\subsubsection{Accuracy}

The delta functions for accuracy are presented in Fig. 4. They show that the Simon effect largely decreased with RT. Interestingly, if we consider the functions for the overall data, we see that for the first quintile the Simon effect was larger in the HST than in the VST, suggesting that automatic response activation was indeed stronger in the HST. With an increasing RT the Simon effect then became negative in the HST, but not in the VST. Consequently, although the functions differ, on average, the Simon effect was similar in both tasks.

We applied a $t$-test on the first quintiles of the overall delta functions for accuracy of the tasks. The analyses revealed a significantly larger congruency effect in the HST compared to the VST $[t=3.19, p<0.01]$, indicating that automatic response activation was stronger in the HST. ${ }^{3}$

We tested the difference in the decline between tasks with another $2 \times 5 \times 2$ ANOVA including the within-participant factors congruency and quintile, and the between-participants factor task. The analysis revealed that the decline was significantly larger in the HST than in the VST $[F(4,132)=9.86, p<0.001]$. However, when testing both tasks separately, there was a reliable two-way interaction between the factors congruency and quintile in the $\operatorname{HST}[F(4,60)=54.23, p<0.001]$ and in the $\operatorname{VST}[F(4,72)=34.94, p<0.001]$, indicating a decreasing Simon effect in accuracy with RT in the VST as well.

\footnotetext{
${ }^{3}$ The variances between the compared groups differed. Thus, we also performed a Welch-test, which does not assume similar variances. The result was the same.
} 

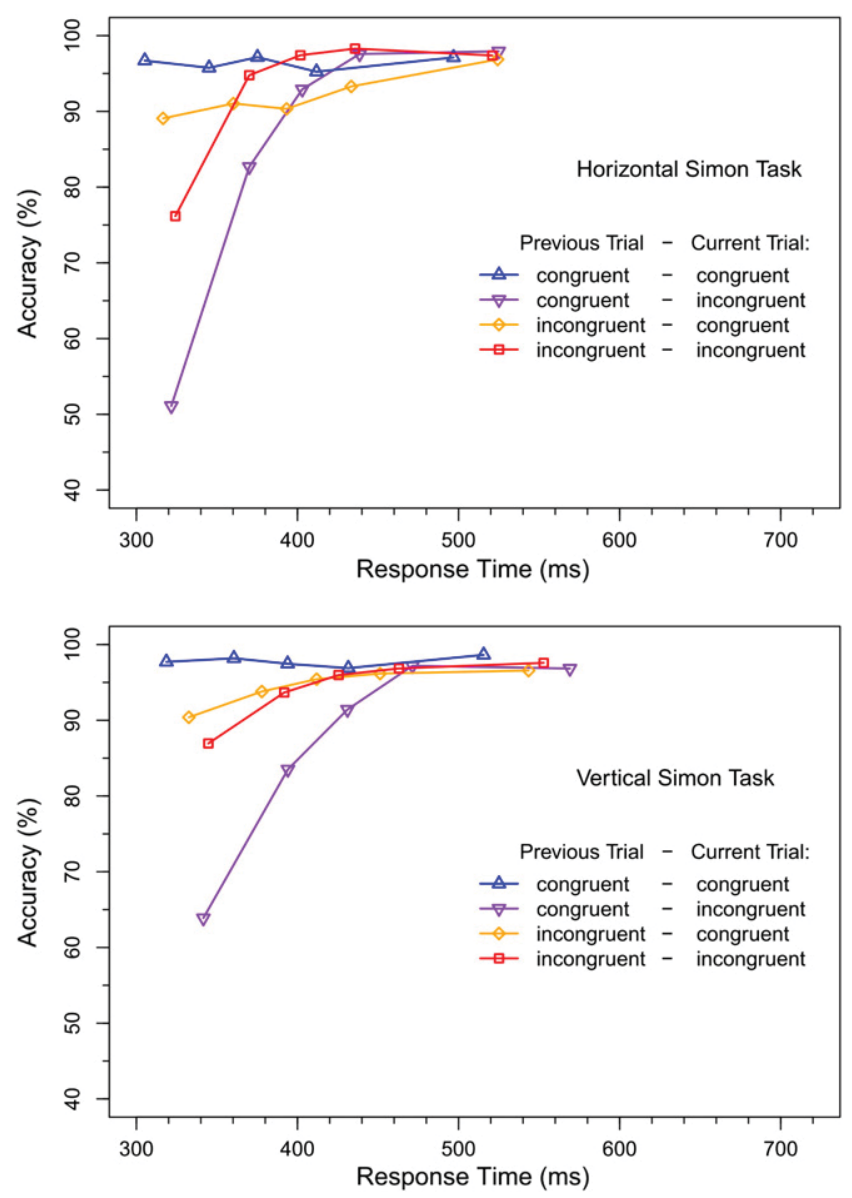

Fig. 3. CAFs for the HST (upper panel) and the VST (lower panel) of Experiment 1. Only the specific functions conditioned on previous-trial congruency are depicted.

Considering the delta functions for accuracy conditioned on previous-trial congruency effects, it is obvious that the decline of the delta function for accuracy is weaker after incongruent trials. This, however, does not necessarily indicate weaker suppression, but seems to be related to the reduced Simon effect in error rates after incongruent trials. The decline in the delta function for accuracy is mainly due to the size of the Simon effect in the first quintile: The larger the effect in the first quintile, the larger the decline of the delta function for accuracy, as the Simon effect in the error rates is quickly decreasing towards zero with slower responses in both tasks. This, however, is mainly related to high response accuracy with slow RT, and relatively independent of suppression of automatic response activation. In the HST it is also obvious that the Simon effect reverses in the second, third, and fourth quintiles after incongruent trials, but not after congruent trials, which clearly indicates strong suppression, although the decline of the delta function for accuracy is rather small. We could not find such a reversal in the VST though.

We tested the differences between the delta functions for accuracy depending on previous-trial congruency in another $2 \times 2 \times 5 \times 2$ ANOVA with the within-participant factors congruency, previous-trial congruency, and quintile, and the between-participants factor task. A significant three-way interaction between congruency, previous-trial congruency, and quintile $[F(4,132)=45.50, p<0.001]$ indicated that the slope differed depending on the previous-trial congruency, that is, the decline was stronger when the previous trial was congruent. The four-way interaction, however, was not significant $[F(4,132)<1]$, indicating that the modulation by the previous-trial congruency did not differ between the tasks.
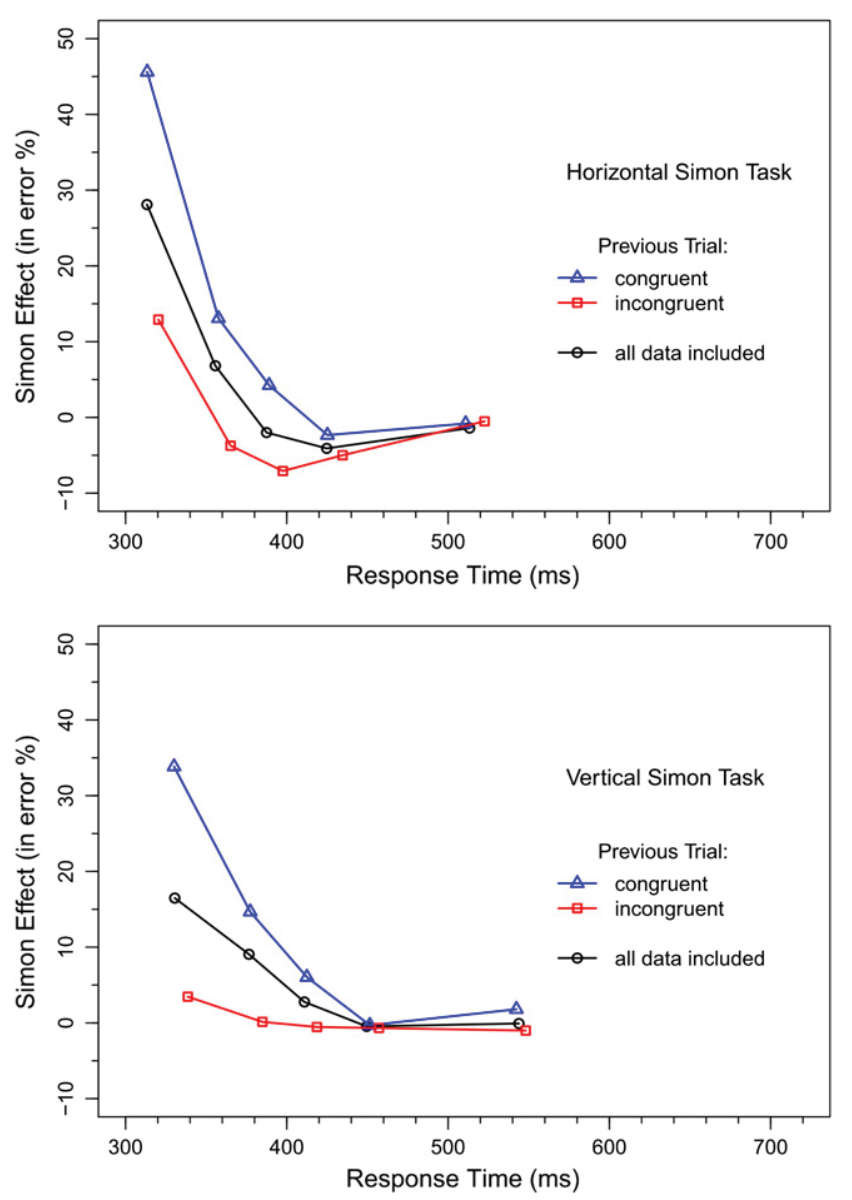

Fig. 4. Delta functions for the accuracy of the HST (upper panel) and the VST (lower panel) of Experiment 1. The overall functions (including all trials) as well as the specific functions conditioned on previous-trial congruency are depicted.

\subsection{Discussion}

Our results show that the mean Simon effect in RT was larger in the VST than in the HST, whereas there was no difference in the error rates, which may have suggested that automatic response activation was stronger in the VST. This interpretation was refuted, however, by the analyses of the delta functions for accuracy, which reflect automatic response activation more directly (Ridderinkhof, 2002b). The analyses show that the congruency effect in accuracy was significantly larger in the first quintile in the HST as compared to the VST, indicating that automatic response activation was indeed stronger in the HST. However, the activation was quickly suppressed, which even produced a negative Simon effect for some quintiles. Together, the larger activation and its stronger suppression in the HST produced an average Simon effect in accuracy that was similar to that in the VST.

If we consider the overall delta functions for RT, then they are in line with former studies (Proctor et al., 2003; Wiegand \& Wascher, 2005; see Proctor et al., 2011 for a review). In the HST the effect decreased with RT, whereas it increased in the VST.

Thus, it seems that automatic response activation and its suppression is stronger in the HST as compared to the VST. This does not indicate that the two tasks differ qualitatively, though. Interestingly, although the Simon effect in the first quintile of the delta function for accuracy was smaller in the VST than in the HST, the pattern was rather similar. For each task the effect decreased significantly. This decrease indicates that fast errors were strongly affected by the irrelevant spatial dimension. Although it cannot be excluded that a cognitive conflict in the stimulus-to-response translation process would have resulted in a 
similar pattern, yet, it seems more likely that automatic response activation also occurred in the VST. This conclusion is further supported by the analyses of the delta functions for RT. If we consider the modulation of suppression by previous-trial congruency, then it is obvious that it was similar in both tasks. After incongruent trials the Simon effect was not only decreased relative to congruent trials, the slope of the delta function for RT was also reduced. The fact that this was the case for both tasks supports the hypothesis that the HST and the VST differ only quantitatively.

The question remains: why was suppression smaller in the VST? Even after incongruent trials we did not observe a negative slope in the delta function for RT, which is usually interpreted as support for the hypothesis that the two tasks differ qualitatively. However, the delta function was flat indicating that the RTs for congruent and incongruent stimuli had a similar variance (Pratte et al., 2010). This is remarkable. Usually, variance increases with mean RT (Wagenmakers \& Brown, 2007), so that one would have expected an increasing delta function with RT. Thus, the fact that this was not the case for the VST after incongruent trials indicates that the variance of RTs for incongruent stimuli was reduced by some mechanism. Most likely this was due to weak suppression.

If there is also suppression of irrelevant activation in the VST, then we should also find declining delta functions. Possibly, the difference in suppression between the HST and the VST depends on the differing amounts of automatic response activation. Thus, if the VST indeed differs only quantitatively from the HST, then it should be possible to further increase suppression. Several variables are conceivable in this respect. In the next experiment the effect of spatial distance between the stimulus locations was investigated.

\section{Experiment 2}

For our reasoning we assumed that the strength of suppression is related to automatic response activation. Consequently, if automatic response activation can be strengthened in a VST, its suppression should increase as well. The delta functions for accuracy in Experiment 1 show that automatic response activation was weaker in the VST than in the HST. Possibly, horizontal and vertical stimulus eccentricities were mentally represented in a different way. One reason could be the cortical magnification factor. Tsai et al. (2013), for instance, reported that the magnification is smaller for vertical distances compared to horizontal ones. Thus, the same distance between stimulus positions could be more dominant in the HST than in the VST. If this is true, one can possibly enhance automatic response activation in the VST by increasing the vertical eccentricity.

To see whether this is indeed the case, we conducted the same VST experiment as in Experiment 1, except that the eccentricity of the stimulus locations was increased. This also offered the possibility of comparing the automatic response activation between the experiments. If a larger vertical eccentricity increases location-induced response activation, then this should also increase suppression of automatic response activation.

\subsection{Method}

18 participants (age range: $19-37$ years, mean: 22.6 years; 5 men) were recruited at the University of Konstanz. All had normal or corrected-to-normal vision and were paid $(8 € / \mathrm{h})$ for their participation or received course credits. Apparatus, stimuli, and procedure were similar to the first experiment, except that only the VST was applied and that the vertical distance between the center and the stimulus positions was increased to $2.4^{\circ}$ of visual angle.

\subsection{Results}

\subsubsection{Response times}

Only correct responses with latencies between $100 \mathrm{~ms}$ and $2000 \mathrm{~ms}$ and following a correct response were entered into data analyses. A twofactor ANOVA with repeated measurements on the factors congruency (congruent or incongruent) and previous-trial congruency (congruent or incongruent) was conducted. The analysis revealed a significant main effect of congruency $[F(1,17)=111.07, p<0.001]$. Participants responded faster on congruent (394 ms) than on incongruent trials $(421 \mathrm{~ms})$. There was also a significant interaction between both factors $[F(1,17)=96.07, p<0.001]$, indicating that the Simon effect was larger after congruent trials ( $55 \mathrm{~ms}$ ) than after incongruent ones $(-3 \mathrm{~ms})$.

For comparison with the VST of Experiment 1 a three-factor ANOVA was conducted, including the within-participant factors congruency and previous-trial congruency, and the between-participants factor eccentricity (large or small; only effects related to the factor eccentricity are reported). There were no significant effects regarding the factor eccentricity $[F(1,35) \leq 1.36]$.

\subsubsection{Error rates}

The mean error rate was 9.73\%. An ANOVA ${ }^{4}$ of the same type as for the response times revealed a significant main effect of congruency $[F(1,17)=62.58, p<0.001$; congruent: $6.36 \%$, incongruent: $13.07 \%]$, and previous-trial congruency $[F(1,17)=22.97, p<0.001$; congruent: $11.24 \%$, incongruent: $8.07 \%$ ]. The interaction between these factors was also significant $[F(1,17)=70.42, p<0.001]$, indicating that the congruency effect was larger after congruent trials (15.42\%) than after incongruent ones $(-2.82 \%)$.

A further three-factor ANOVA with the within-participant factors congruency and previous-trial congruency, and the between-participants factor eccentricity was conducted for comparison with Experiment 1. There was a significant main effect on the factor eccentricity $[F(1,35)=8.38$, $p<0.01$ ], meaning that the participants in the experiment with a large vertical eccentricity responded less accurately $(9.73 \%)$ than those in the experiment with a small vertical eccentricity $(6.55 \%)$. Also the threeway interaction reached significance $[F(1,35)=4.58, p<0.05]$, indicating that the modulation of the Simon effect by previous-trial congruency was stronger for the increased vertical eccentricity.

\subsubsection{Distributional analyses}

3.2.3.1. Response times. The CDFs conditioned on previous-trial congruency are depicted in Fig. 5 (upper panel), and the delta functions for RT are shown in Fig. 6 (upper panel). A $2 \times 5$ ANOVA $^{5}$ with repeated measurements including the factors congruency (congruent, incongruent) and quintile ( 1 to 5 ) revealed that the two factors interacted significantly $[F(4,68)=7.47, p<0.001]$, indicating an increasing delta function for RT. If we take the factor previous-trial congruency (congruent, incongruent) into account, then there was a significant three-way interaction between congruency, quintile, and previous-trial congruency $[F(4,68)=8.66, p<0.001]$.

The individual previous-trial congruency conditions were also analyzed separately. For responses after congruent trials there was a significant two-way interaction $[F(4,68)=6.83, p<0.001]$, which indicates that the Simon effect increased with RT (see Fig. 6, upper panel). Most importantly, the two-way interaction for responses after incongruent trials was also significant $[F(4,68)=5.47, p<0.001]$. In this case the interaction indicates that the Simon effect decreased with RT.

A three-factor ANOVA was conducted, for the comparison with the VST of Experiment 1, including the within-participant factors congruency

\footnotetext{
${ }^{4}$ The analyses with arcsine transformed error rates again revealed the same essential results.

${ }^{5}$ Similar to Experiment 1, comparing the slopes of the delta functions for RT, estimated by least square regression (Pratte et al., 2010), leads to the same essential results.
} 

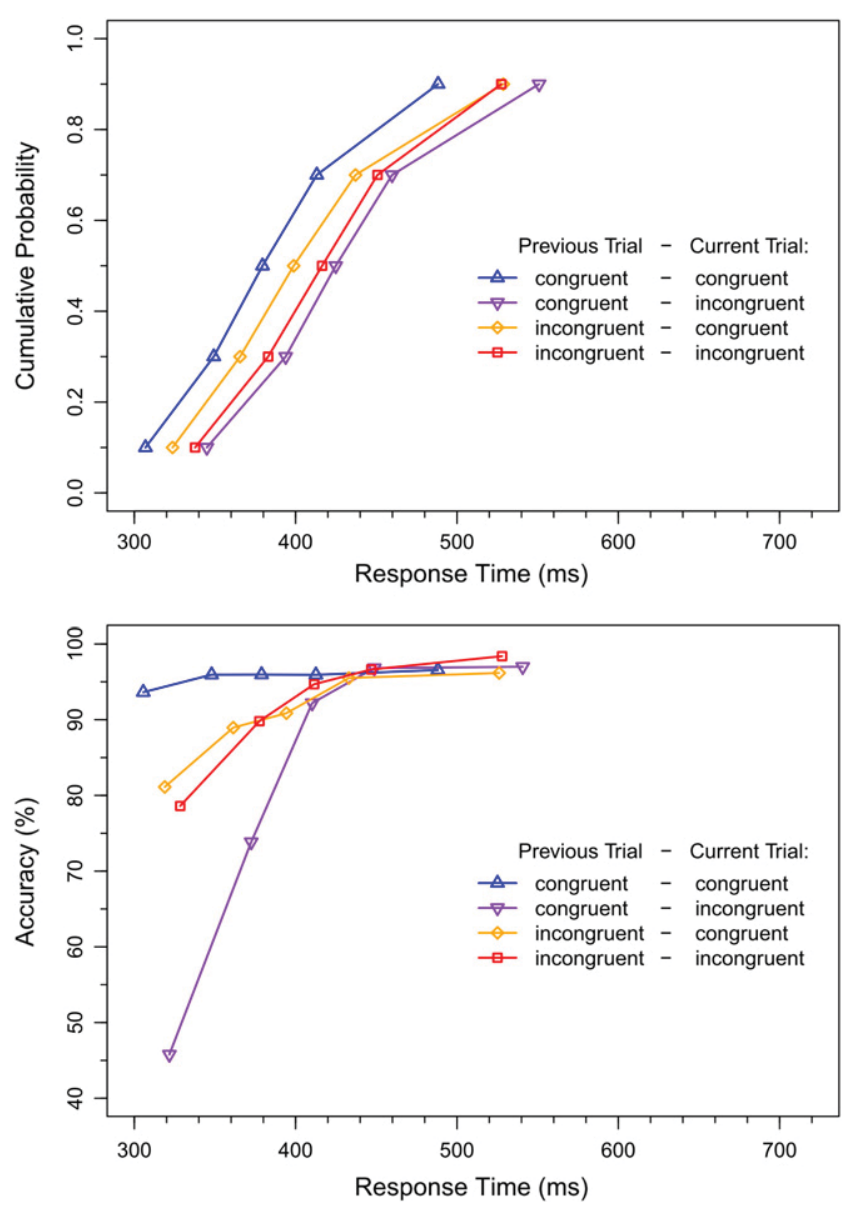

Fig. 5. CDF (upper panel) and CAF (lower panel) of the VST in Experiment 2. Only the specific functions conditioned on previous-trial congruency are depicted.

and quintile, and the between-participants factor eccentricity (large or small). There were no significant effects regarding the factor eccentricity. This also held for further analyses involving previous-trial congruency.

3.2.3.2. Accuracy. Fig. 5 (lower panel) presents the CAFs conditioned on previous-trial congruency and Fig. 6 (lower panel) shows the corresponding delta functions. A similar analysis as for the effects in RT was conducted. As expected, the Simon effect was large for fast responses and then decreased significantly with $\operatorname{RT}[F(4,68)=96.07, p<0.001]$. The effect also varied with previous-trial congruency $[F(4,68)=24.69$, $p<0.001]$. Separate analyses of the Simon effect for the individual previous-trial congruency conditions revealed a significant decline after congruent trials $[F(4,68)=56.30, p<0.001]$, but no such modulation after incongruent trials $[F(4,68)<1]$, which, however, is again due to the small mean Simon effect.

For comparison of automatic response activation between the VSTs in both experiments, we conducted a $t$-test on the first quintiles of the overall delta functions for accuracy of the tasks. ${ }^{6}$ It revealed a significant larger congruency effect with large stimulus eccentricity $[t=2.32, p<0.05]$, indicating that automatic response activation was stronger in Experiment 2 as compared to the VST with a smaller eccentricity in Experiment 1.

A further three-factor ANOVA for the comparison with the VST in Experiment 1 with the within-participant factors congruency and quintile, and the between-participants factor eccentricity revealed that there was a significant interaction between the factors eccentricity and quintile $[F(4,140)=12.20, p<0.01]$, indicating a stronger decrease in the error

\footnotetext{
${ }^{6}$ The variances between the compared groups again differed. Therefore, we performed another Welch-test (not assuming similar variances). The result remained the same.
}
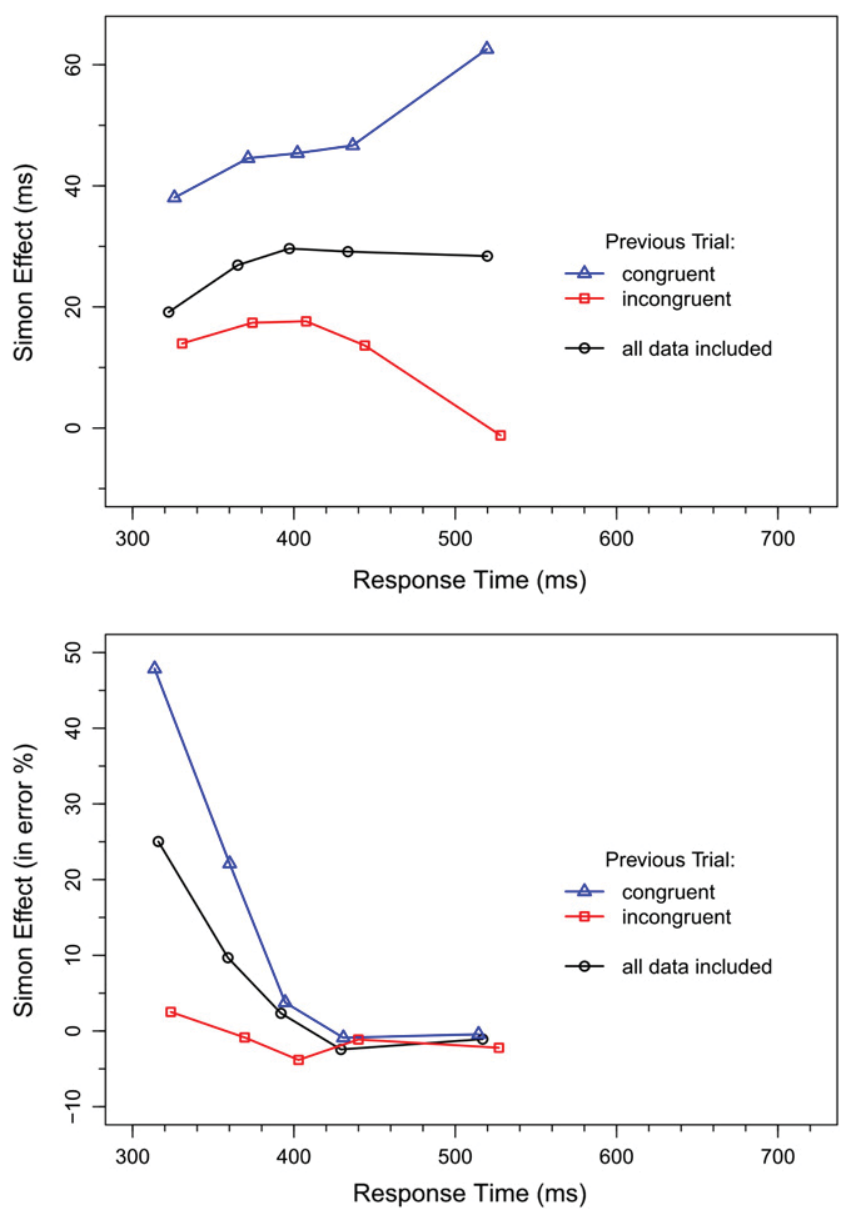

Fig. 6. Delta functions for the latencies of correct responses (upper panel) and for accuracy (lower panel) of the VST in Experiment 2.

rates across the quintiles with larger vertical eccentricity. Also the threeway interaction reached significance $[F(4,140)=4.05, p<0.01]$, indicating that there was a stronger decline in the delta function for accuracy in Experiment 2. These effects are again due to the generally stronger Simon effect in the first quintile with increased vertical eccentricity.

Regarding the previous-trial congruency, there was only a marginally significant four-way interaction $[F(4,140)=2.28, p=0.06]$. It might indicate that the influence of the previous-trial congruency on the slope of the delta function is stronger in the task with larger vertical eccentricity.

\subsection{Discussion}

Despite the increased vertical eccentricity of the stimuli, the mean Simon effect in RT was of similar size as in the previous experiment. In the error rates, however, the Simon effect was larger and the modulation of the Simon effect by previous-trial congruency was stronger. Importantly, automatic response activation - as indicated by the first quintile in the delta functions for accuracy - was stronger in the present experiment than in the VST of Experiment 1.

If we consider the overall delta function for the RT (Fig. 6, upper panel), we see that the Simon effect first increased with RT but then leveled off. Importantly, there was again a modulation of the slope of the delta function for RT by previous-trial congruency. If the previous trial was incongruent, the Simon effect decreased substantially for the slower responses, indicating suppression of automatic response activation.

Together, the results of our second experiment demonstrate that response suppression can also occur in the VST and is not restricted to 
the HST. Moreover, although suppression seems to occur more naturally or easily in the HST, the observed negatively sloped delta function for RT supports the hypothesis that the HST and the VST differ only quantitatively.

\section{General discussion}

In the present study we examined whether the VST differs qualitatively or quantitatively from the HST in automatic response activation and its suppression. A characteristic of the HST is that the Simon effect usually decreases with RT, which has been interpreted as an indication of an irrelevant location-induced activation that is increasingly suppressed (e.g., Ridderinkhof et al., 2004). Because such a decrease was rarely observed for the VST, it has been proposed that automatic response activation and, consequently, its suppression occurs only in the HST, but not in the VST. It is, however, possible that location-induced activation merely differs quantitatively between the tasks. Perhaps locations along the vertical meridian activate a corresponding response to a lesser extent than locations along the horizontal meridian and, therefore, the suppression of automatic response activation is also weaker (Rubichi et al., 2005). Thus, if the inhibitory demand is indeed lower in the VST, then it is no wonder that little or no suppression has been observed in the VST.

However, the degree of suppression does not only depend on the amount of response activation, but also on previous-trial congruency. Suppression is usually stronger after an incongruent trial than after a congruent one in the HST (Ridderinkhof, 2002a). This is also reflected by the delta functions for RT and for accuracy. If response activation and its suppression differ only quantitatively between the tasks, a similar modulation of the Simon effect should be found for the VST. A first hint in this direction has been given by Stürmer et al. (2002). They showed descriptively that, after incongruent trials, the Simon effect was not only generally reduced, but also that the delta function for RT was slightly decreasing. The authors did not test this effect for significance, however. Therefore, in the present study this issue was investigated again by directly comparing the performance in a HST with that in a VST. Moreover, automatic response activation was compared between the tasks using delta functions for accuracy.

As expected, automatic response activation was stronger in the HST, compared to the VST. Also, the analyses of the delta functions for RT were in line with previous results (see Proctor et al., 2011 for a review). The Simon effect decreased with RT in the HST, but not in the VST. More specifically, in the HST the delta function for RT was negatively sloped, whereas it was positively sloped in the VST. As a consequence of the lower suppression, the Simon effect was larger in the VST than in the HST.

In support of the quantitative-difference hypothesis, though, we found a comparable response activation pattern in the delta functions for accuracy and a similar modulation of the Simon effect by previoustrial congruency in the delta functions for RT in the VST and in the HST. Moreover, the delta function for responses after an incongruent trial had a smaller slope, compared to that for responses after congruent trials, similar to the HST in Ridderinkhof (2002a). Although this result is in line with the idea that the two versions of the Simon task merely differ quantitatively, we did not observe a negatively sloped delta function for RT in the VST, even after incongruent trials. Flat delta functions for RT have often been interpreted in the sense that there was no response suppression.

Therefore, in our second experiment we tried to increase the inhibitory demand in the VST by increasing the vertical eccentricity of the stimulus positions. As a result, the Simon effect in RT substantially decreased with RT, at least for responses after incongruent trials, which is a clear indication of suppression of automatic response activation, and, therefore, supports the quantitative-difference hypothesis. Also the degree of automatic response activation was enhanced relative to the VST in Experiment 1. This suggests that suppression depends on the strength of automatic response activation.

Interestingly, in all three versions of the Simon task, suppression was increased after incongruent as compared to congruent trials and, as predicted by conflict adaptation theories (e.g., Stürmer et al., 2002), automatic response activation was lower as well. This observation seems to violate our idea that suppression of the automatically induced response is positively related to the strength of automatic response activation. However, if we assume that suppression of irrelevant activation is related to the amount of automatic response activation, this does not imply that suppression is directly induced by automatic response activation in every case. Quite the contrary, we would not expect that at all. First, it has been shown that suppression is rather flexible and can be adjusted according to the demands of the task (Hübner \& Mishra, 2013), arguing against a purely reflexive nature. Second, it has previously been shown in the HST that suppression increases after incongruent predecessor trials, although automatic response activation was weaker (Ridderinkhof, 2002a). Ridderinkhof (2002a) suggested that "the presence of incorrect activation on a preceding [incongruent] trial might tune up the level of inhibitory control on the subsequent event" (p. 514). Most likely, the amount of incorrect activation on the previous trial also has an impact on the inhibitory control on the subsequent event. Conclusively, we claim that suppression is related to the amount of automatic response activation in the task generally, and especially to that on the preceding trial.

The observation that delta functions for RT in the VST constantly increased, led to the idea that the HST and VST differ qualitatively with respect to irrelevant response activation (e.g., Wascher et al., 2001). Our result of a declining delta function challenges this hypothesis, although we cannot generally conclude that automatic response activation is similar in both tasks. Suppression of the irrelevant response does not necessarily imply automatic response activation via the unconditional route. Indeed, there is also other evidence for a difference in response activation between the tasks (Vallesi et al., 2005; Vu, 2007).

Nevertheless, our delta functions for accuracy support the idea of a merely quantitative difference between the tasks. Although in Experiment 1 the Simon effect in accuracy was weaker for the VST than for the HST, the corresponding delta functions also decreased, indicating that fast errors were strongly influenced by the irrelevant stimulus dimension. This pattern is very characteristic of automatic response activation. Whether a quantitative difference between the tasks is sufficient to explain previous results is subject to future research. That similar mechanisms are at play in both tasks is further supported by a study using a different conflict paradigm, the Eriksen flanker task (Eriksen \& Eriksen, 1974). Burle, Spieser, Servant, and Hasbroucq (2013) have shown that suppression can also occur in the flanker task, suggesting that suppression is by no means restricted to the horizontal version of the Simon task.

The fact that response suppression in the VST takes place mainly after incongruent trials, which usually accounts for half of all trials, explains why the overall delta functions for RT are not declining with RT. An exception seems to be difficult VSTs, e.g., where the stimulusresponse mapping varies randomly (Wiegand \& Wascher, 2007). Obviously, a greater task difficulty can also lead to a higher inhibitory demand.

Taken together, the present study demonstrates that response suppression can also occur in a VST. Moreover, in a VST and a HST we found a similar modulation of the Simon effect by previous-trial congruency. These results indicate a quantitative rather than a qualitative difference between the two tasks.

\section{Acknowledgments}

We thank Michael Dambacher, Shreyasi Mishra, and the two anonymous reviewers for their valuable comments concerning this paper. 


\section{References}

Botvinick, M. M., Braver, T. S., Barch, D.M., Carter, C. S., \& Cohen, J.D. (2001). Conflict monitoring and cognitive control. Psychological Review, 108, 624-652.

Buetti, S., \& Kerzel, D. (2008). Time course of the Simon effect in pointing movements for horizontal, vertical, and acoustic stimuli: Evidence for a common mechanism. Acta Psychologica, 129, 420

Burle, B., Spieser, L., Servant, M. \& Hasbroucq, T. (2013). Distributional reaction time properties in the Eriksen task: Marked differences or hidden similarities with the Simon task? Psychonomic Bulletin \& Review, 1-8.

De Jong, R., Liang, C. C., \& Lauber, E. (1994). Conditional and unconditional automaticity: A dual-process model of effects of spatial stimulus-response correspondence. Journal of Experimental Psychology: Human Perception and Performance, 20, 731.

Egner, T. (2007). Congruency sequence effects and cognitive control. Cognitive, Affective, $\mathcal{E}$ Behavioral Neuroscience, 7, 380-390.

Eriksen, B.A., \& Eriksen, C. W. (1974). Effects of noise letters upon the identification of a target letter in a nonsearch task. Perception \& Psychophysics, 16, 143-149.

Hommel, B. (2004). Event files: Feature binding in and across perception and action. Trends in Cognitive Sciences, 8, 494-500.

Hommel, B. (2011). The Simon effect as tool and heuristic. Acta Psychologica, 136, 189-202.

Hübner, R., \& Mishra, S. (2013). Evidence for strategic suppression of irrelevant activation in the Simon task. Acta Psychologica, 144, 166-172.

Kornblum, S., Hasbroucq, T., \& Osman, A. (1990). Dimensional overlap: Cognitive basis for stimulus-response compatibility - A model and taxonomy. Psychological Review, 97 , 253-270.

Mayr, U., Awh, E., \& Laurey, P. (2003). Conflict adaption effects in the absence of executive control. Nature Neuroscience, 6, 450-452.

Pratte, Rouder, J., Morey, R., \& Feng, C. (2010). Exploring the differences in distributional properties between Stroop and Simon effects using delta plots. Attention, Perception, E Psychophysics, 72, 2013-2025.

Proctor, R. W., Miles, J.D., \& Baroni, G. (2011). Reaction time distribution analysis of spatial correspondence effects. Psychonomic Bulletin \& Review, 18, 242-266.

Proctor, R. W., \& Vu, K. P. L. (2010). Stimulus-response compatibility for mixed mappings and tasks with unique responses. The Quarterly Journal of Experimental Psychology, 63, 320-340.

Proctor, R. W., Vu, K. P. L., \& Nicoletti, R. (2003). Does right-left prevalence occur for the Simon effect? Attention, Perception, E' Psychophysics, 65, 1318-1329.

Ratcliff, R. (1979). Group reaction time distributions and an analysis of distribution statistics. Psychological Bulletin, 86, 446-461.

Ridderinkhof, K. R. (2002a). Activation and suppression in conflict tasks: Empirical clarification through distributional analyses. In W. Prinz, \& B. Hommel (Eds.), Common Mechanisms in Perception and Action. Attention E Performance, vol. XIX. (pp. 494-519). Oxford: Oxford University Press.
Ridderinkhof, K. R. (2002b). Micro- and macro-adjustments of task set: Activation and suppression in conflict tasks. Psychological Research, 66, 312-323.

Ridderinkhof, K. R., van den Wildenberg, W. P.M., Wijnen, J., \& Burle, B. (2004). Response inhibition in conflict tasks is revealed in delta plots. Cognitive Neuroscience of Attention, 369-377.

Rubichi, S., Nicoletti, R., \& Umiltà, C. (2005). Right-left prevalence with task-irrelevant spatial codes. Psychological Research, 69, 167-178.

Simon, J. R., \& Rudell, A. P. (1967). Auditory SR compatibility: The effect of an irrelevant cue on information processing. Journal of Applied Psychology, 51, 300.

Stürmer, B., \& Leuthold, H. (2003). Control over response priming in visuomotor processing: A lateralized event-related potential study. Experimental Brain Research, 153, $35-44$.

Stürmer, B., Leuthold, H., Soetens, E., Schröter, H., \& Sommer, W. (2002). Control over location-based response activation in the Simon task: Behavioral and electrophysiological evidence. Journal of Experimental Psychology: Human Perception and Performance, 28, 1345.

Tsai, L. T., Chen, C. C., Jang, Y., \& Liao, K. M. (2013). The meridian effect on the cortical magnification factor for visual word form identification. Paper presented at the Vision Sciences Society 13th Annual Meeting, Florida, USA.

Vallesi, A., Mapelli, D., Schiff, S., Amodio, P., \& Umilta, C. (2005). Horizontal and vertical Simon effect: Different underlying mechanisms? Cognition, 96, B33-B43.

Vallesi, A., \& Umiltà, C. A. (2009). Decay of stimulus spatial code in horizontal and vertical Simon tasks. The Journal of General Psychology: Experimental, Psychological, and Comparative Psychology, 136, 350-373.

Vu, K. -P. L. (2007). Influences on the Simon effect of prior practice with spatially incompatible mappings: Transfer within and between horizontal and vertical dimensions. Memory \& Cognition, 35, 1463-1471.

Wagenmakers, E. J., \& Brown, S. (2007). On the linear relation between the mean and the standard deviation of a response time distribution. Psychological Review, 114, 830.

Wascher, E., Schatz, U., Kuder, T., \& Verleger, R. (2001). Validity and boundary conditions of automatic response activation in the Simon task. Journal of Experimental Psychology: Human Perception and Performance, 27, 731-751.

Wiegand, K., \& Wascher, E. (2005). Dynamic aspects of stimulus-response correspondence: Evidence for two mechanisms involved in the Simon effect. Journal of Experimental Psychology: Human Perception and Performance, 31, 453-464.

Wiegand, K., \& Wascher, E. (2007). The Simon effect for vertical S-R relations: Changing the mechanism by randomly varying the S-R mapping rule? Psychological Research $71,219-233$.

Wijnen, J. G., \& Ridderinkhof, K. R. (2007). Response inhibition in motor and oculomotor conflict tasks: different mechanisms, different dynamics? Brain and Cognition, 63, 260-270. 